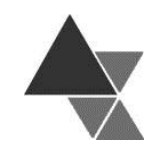

\title{
Brasil, 2013: mais segurança alimentar
}

\author{
Rodolfo Hoffmann ${ }^{1}$
}

A divulgação, em 2014, dos dados da Pesquisa Nacional por Amostra de Domicílios (PNAD) de 2013, incluindo a pesquisa suplementar sobre segurança alimentar, permite atualizar as informações sobre esse tema relevante e analisar a evolução da segurança alimentar no País, comparando esses dados com os obtidos nas PNADs de 2004 e 2009. É clara a tendência de redução da insegurança alimentar. O ajuste de modelos de lógite para os dados de 2013 leva a reafirmar que os determinantes fundamentais da segurança alimentar de um domicílio são a renda domiciliar per capita, a escolaridade, a disponibilidade de água encanada, luz elétrica e esgoto apropriado e a estabilidade da renda (o emprego com carteira é mais favorável à segurança alimentar do que o emprego sem carteira ou o trabalho por conta própria). Modelos de lógite estimados agregando os dados de 2009 e 2013 permitem constatar que há parcela da redução da insegurança alimentar associada a mudanças que não foram apropriadamente captadas pelas variáveis explanatórias consideradas. Uma dessas mudanças favoráveis provavelmente é a redução na desigualdade da distribuição da renda. São, ainda, analisadas as frequências das respostas à pergunta sobre a atitude adotada quando faltou alimento.

Palavras-chave: segurança alimentar, modelos de lógite, Brasil.

\section{Brazil, 2013: improved food security}

The data from the 2013 Brazilian National Household Survey (PNAD) became available in 2014. This survey included a supplementary questionnaire on food security, which was also applied in the surveys of 2004 and 2009. Thus, it is possible to update the analysis of food insecurity in Brazil and examine its evolution since 2004. The results show a clear reduction in food insecurity. The estimated logit models for 2013 confirm that the main determinants of food security are the per capita household income, schooling of the household head, existence of electric light, piped water, adequate sewage and stability of the income source. A logit model estimated by pooling the 2009 and 2013 data shows that a part of the reduction in food insecurity observed in this period cannot be attributed to changes in the set of explanatory variables included. One possible explanation is that the model does not capture certain changes in income distribution that might have had a positive impact on food security. The answers to new questions introduced in 2013, about the actions taken by the household when suffering lack of food, are also analyzed.

Key-words: food security, logit models, Brazil.

1 Professor Sênior da Escola Superior de Agricultura "Luiz de Queiroz" (ESALQ), Universidade de São Paulo (USP), com apoio do CNPq. O autor agradece a Angela Kageyama e a Josimar Gonçalves de Jesus pela leitura crítica de uma versão preliminar do artigo. Correspondência: ESALQ-LES, Cx. Postal 9, CEP 13418-900. Piracicaba, SP. E-mail: hoffmannr@ usp.br. 


\section{INTRODUÇÃO}

A Pesquisa Nacional por amostra de Domicílios (PNAD) de 2013 foi a terceira na qual o IBGE incluiu a aplicação da Escala Brasileira de Insegurança Alimentar (EBIA). Essa pesquisa já havia sido incluída na PNAD em 2004 e 2009. Os novos dados, divulgados em 2014, permitem atualizar as análises elaboradas anteriormente [1,2] e abordar novos aspectos do tema.

$\mathrm{Na}$ PNAD de 2013 a aplicação da EBIA foi baseada nas respostas, para cada domicílio da amostra, às 14 perguntas apresentadas no Quadro 1.

Quadro 1. Perguntas da Escala Brasileira de Insegurança Alimentar usadas em 2013.

\begin{tabular}{|c|c|}
\hline 1 & $\begin{array}{l}\text { Nos últimos três meses, os moradores desse domicílio tiveram a preocupação de que os alimentos acabassem antes } \\
\text { de poderem comprar mais comida? }\end{array}$ \\
\hline 2 & $\begin{array}{l}\text { Nos últimos três meses, os alimentos acabaram antes que os moradores desse domicílio tivessem dinheiro para } \\
\text { comprar mais comida? }\end{array}$ \\
\hline 3 & $\begin{array}{l}\text { Nos últimos três meses, os moradores desse domicílio ficaram sem dinheiro para ter uma alimentação saudável e } \\
\text { variada? }\end{array}$ \\
\hline 4 & $\begin{array}{l}\text { Nos últimos três meses os moradores desse domicílio comeram apenas alguns alimentos que ainda tinham porque } \\
\text { o dinheiro acabou? }\end{array}$ \\
\hline 5 & $\begin{array}{l}\text { Nos últimos três meses, algum morador de } 18 \text { anos ou mais de idade deixou de fazer alguma refeição porque não } \\
\text { havia dinheiro para comprar comida? }\end{array}$ \\
\hline 6 & $\begin{array}{l}\text { Nos últimos três meses, algum morador de } 18 \text { anos ou mais de idade, alguma vez, comeu menos do que achou } \\
\text { que devia porque não havia dinheiro para comprar comida? }\end{array}$ \\
\hline 7 & $\begin{array}{l}\text { Nos últimos três meses, algum morador de } 18 \text { anos ou mais de idade, alguma vez, sentiu fome, mas não comeu } \\
\text { porque não havia dinheiro para comprar comida? }\end{array}$ \\
\hline 8 & $\begin{array}{l}\text { Nos últimos três meses, algum morador de } 18 \text { anos ou mais de idade, alguma vez, fez apenas uma refeição ao dia } \\
\text { ou ficou um dia inteiro sem comer porque não tinha dinheiro para comprar comida? }\end{array}$ \\
\hline 9 & $\begin{array}{l}\text { Nos últimos três meses, algum morador com menos de } 18 \text { anos de idade, alguma vez, deixou de ter uma } \\
\text { alimentação saudável e variada porque não havia dinheiro para comprar comida? }\end{array}$ \\
\hline 10 & $\begin{array}{l}\text { Nos últimos três meses, algum morador com menos de } 18 \text { anos de idade, alguma vez, não comeu quantidade } \\
\text { suficiente de comida porque não havia dinheiro para comprar comida? }\end{array}$ \\
\hline 11 & $\begin{array}{l}\text { Nos últimos três meses, alguma vez, foi diminuída a quantidade de alimentos das refeições de algum morador com } \\
\text { menos de } 18 \text { anos de idade, porque não havia dinheiro para comprar comida? }\end{array}$ \\
\hline 12 & $\begin{array}{l}\text { Nos últimos três meses, alguma vez, algum morador com menos de } 18 \text { anos de idade deixou de fazer alguma } \\
\text { refeição porque não havia dinheiro para comprar a comida? }\end{array}$ \\
\hline 13 & $\begin{array}{l}\text { Nos últimos três meses, alguma vez, algum morador com menos de } 18 \text { anos de idade sentiu fome, mas não comeu } \\
\text { porque não havia dinheiro para comprar comida? }\end{array}$ \\
\hline 14 & $\begin{array}{l}\text { Nos últimos três meses, alguma vez, algum morador com menos de } 18 \text { anos de idade fez apenas uma refeição ao } \\
\text { dia ou ficou sem comer por um dia inteiro porque não havia dinheiro para comprar comida? }\end{array}$ \\
\hline
\end{tabular}


O domicílio foi classificado em uma de quatro categorias, conforme o número de respostas "sim" e a presença ou não de morador com menos de 18 anos de idade, como mostra a Tabela 1.

Tabela 1. Pontuação para classificação dos domicílios, com e sem menores de 18 anos de idade.

\begin{tabular}{l|c|c}
\hline \multirow{2}{*}{\multicolumn{1}{c|}{ Classificação }} & \multicolumn{2}{c}{ Número de respostas "sim" para domicílios } \\
\cline { 2 - 3 } & Com menores de 18 anos & Sem menores de 18 anos \\
\hline Segurança alimentar & 0 & 0 \\
Insegurança alimentar leve & 1 a 5 & 1 a 3 \\
Insegurança alimentar moderada & 6 a 9 & 4 a 5 \\
Insegurança alimentar grave & 10 a 14 & 6 a 8 \\
\hline
\end{tabular}

Fonte: IBGE, Diretoria de Pesquisas, Coordenação de Trabalho e Rendimento [3].

Uma boa exposição sobre a origem e evolução da EBIA pode ser encontrada nas publicações do IBGE sobre o tema [3,4,5]. Segall-Corrêa e Marin-Leon [6] apresentam um breve histórico e uma defesa da relevância da escala.

O exame do conjunto de perguntas apresentadas no Quadro 1 deixa claro que a EBIA avalia, essencialmente, a segurança alimentar (SA) food security), isto é, o acesso aos alimentos. A menção, repetida, à falta de "dinheiro para comprar comida" se justifica porque no Brasil o acesso aos alimentos se dá, geralmente, por meio da compra.

A EBIA não avalia apropriadamente a segurança dos alimentos (food safety), uma vez que se baseia em respostas de consumidores que não têm acesso a laboratórios de análises químicas e microbiológicas ${ }^{2}$. Cabe aos órgãos da vigilância sanitária fiscalizar a produção de alimentos e a eventual existência de diversos tipos de contaminações dos alimentos comercializados.

É claro que a EBIA não permite avaliar, adequadamente, a Segurança Alimentar e Nutricional (SAN), que é algo bem mais abrangente que a Segurança Alimentar stricto sensu, incluindo também a segurança dos alimentos (qualidade sanitária e biológica dos alimentos), as condições de saúde dos consumidores e até a sustentabilidade da produção dos alimentos, com conservação da biodiversidade (IBGE, $2014{ }^{(3)}$.

Cabe ressaltar o caráter subjetivo de algumas perguntas apresentadas no Quadro 1. $\mathrm{Na}$ primeira questiona-se sobre a "preocupação" dos moradores. $\mathrm{Na}$ sexta pergunta há referência a "comeu menos do que achou que devia". Isso aumenta o grau de vagueza da medida. Mas certo grau de vagueza existe em muitos conceitos. Pobreza é, tipicamente, um conceito bastante vago, mas isso não reduz a importância de medi-la. A informação de que "ficou sem comer por um dia inteiro" é mais objetiva, mas é óbvia a necessidade de perguntas com caráter subjetivo para construir a escala de insegurança alimentar. $\mathrm{O}$ reconhecimento do caráter parcialmente subjetivo da EBIA é importante para entender alguns fenômenos que serão analisados adiante.

\section{A redução da insegurança alimentar de 2004 a 2013}

Tomando o cuidado de refazer a classificação dos domicílios na PNAD de 2004, utilizando as respostas de apenas 14 perguntas, como está explicado no Apêndice A, obtivemos os resultados apresentados na Tabela 2 , já divulgados pelo IBGE [3].

2 Perez-Escamilla e Segall-Corrêa [] (p. 18) afirmam, explicitamente, que as escalas

baseadas em experiências pessoais não captam a food safety dimension. 
Tabela 2. Porcentagem de domicílios particulares e de moradores conforme categorias de segurança alimentar. Brasil: 2004, 2009 e 2013.

\begin{tabular}{l|r|r|r|r|r|r}
\hline \multirow{2}{*}{ Categorias de SA } & \multicolumn{3}{c|}{ Porcentagem de domicílios } & \multicolumn{3}{c}{ Porcentagem de moradores } \\
\cline { 2 - 7 } & 2004 & 2009 & 2013 & 2004 & 2009 & 2013 \\
\hline Com SA & 65,1 & 69,8 & 77,4 & 60,1 & 65,9 & 74,2 \\
Insegurança leve & 18,0 & 18,7 & 14,8 & 20,3 & 20,9 & 17,1 \\
Insegurança moderada & 9,9 & 6,5 & 4,6 & 11,3 & 7,4 & 5,1 \\
Insegurança grave & 6,9 & 5,0 & 3,2 & 8,2 & 5,8 & 3,6 \\
\hline Fonte: IBGE, 2014 [3]. &
\end{tabular}

A proporção de moradores com segurança alimentar é sempre menor que a correspondente proporção de domicilios e ocorre o contrário para as porcentagens de moradores e domicilios com insegurança alimentar leve, moderada ou grave, pois os domicílios com segurança alimentar possuem, em média, menos pessoas. Em 2013 o número médio de moradores por domicílio particular é 3,09 no geral, 2,96 para domicílios com segurança alimentar, 3,57 para os com insegurança leve, 3,46 para os com insegurança moderada e 3,43 para domicílios particulares com insegurança alimentar grave. No geral esse número caiu de 3,52 em 2004 para 3,09 em 2013, ocorrendo redução nas 4 categorias de $\mathrm{SA}$, mais intensa no caso dos domicílios com insegurança alimentar moderada ou grave. Nessa última categoria o tamanho médio do domicílio caiu de 4,14 em 2004 para 3,43 em 2013.

Tanto para domicílios como para moradores, a Tabela 2 mostra, em geral, o aumento da proporção com segurança alimentar e a redução da proporção nos 3 níveis de insegurança alimentar. A única exceção é o ligeiro crescimento da incidência de insegurança leve entre 2004 e 2009, explicável pelo caráter subjetivo de algumas perguntas da EBIA e pela disseminação da ideia de que a alimentação adequada é um direito humano (e um dever do Estado).

Comparando as proporções em 2013 com as de anos anteriores, pode-se afirmar, sempre, que há menos insegurança alimentar em 2013. Para insegurança moderada e grave isso é verdade em todas as 5 grandes regiões do País (ver IBGE, 2014 [3], Tabela 4).

A Tabela 3 mostra a evolução das porcentagens de domicilios particulares nas categorias de SA para áreas urbanas e áreas rurais.

Tabela 3. Porcentagem de domicílios particulares, conforme situação urbana ou rural, nas 4 categorias de SA. Brasil: 2004, 2009 e 2013.

\begin{tabular}{l|r|r|r|r|r|r}
\hline \multirow{2}{*}{ Categorias de AS } & \multicolumn{3}{c|}{ Domicílios urbanos } & \multicolumn{3}{c}{ Domicílios rurais } \\
\cline { 2 - 7 } & 2004 & 2009 & 2013 & 2004 & 2009 & 2013 \\
\hline Com segurança alimentar & 66,7 & 70,7 & 79,5 & 56,2 & 64,8 & 64,7 \\
Insegurança leve & 17,6 & 18,5 & 13,7 & 20,2 & 19,5 & 21,4 \\
Insegurança moderada & 9,2 & 6,1 & 3,9 & 14,0 & 8,5 & 8,4 \\
Insegurança grave & 6,5 & 4,6 & 2,8 & 9,6 & 7,1 & 5,5 \\
\hline Fonte: IBGE, 2014 [3]. &
\end{tabular}


Chama a atenção o fato de a proporção de domicilios rurais com segurança alimentar não ter crescido de 2009 a 2013. Mas é necessário lembrar que a delimitação das áreas urbanas e rurais adotada pelo IBGE foi alterada por ocasião do Censo Demográfico de 2010. Ao fazer isso o IBGE respeita a legislação municipal vigente na ocasião. Trata-se, portanto, de uma delimitação de caráter político-administrativo, sem embasamento socioeconômico. A tendência geral é de ampliar as áreas classificadas como urbanas e restringir as rurais. $\mathrm{O}$ rural mais restrito tende a ficar com domicílios relativamente mais pobres. É possível, então, que a evolução pouco favorável da SA nos domicílios rurais de 2009 a 2013 seja devida, pelo menos em parte, ao fato de o "rural" de 2013 ser uma área mais restrita cujos domicílios são, em geral, relativamente pobres.

Uma alternativa é destacar os domicílios agrícolas dos demais, definindo como agrícola o domicílio cuja pessoa de referência tem atividade principal no setor agrícola, como é feito na Tabela 4.

Tabela 4. Porcentagem de domićlios particulares nas 4 categorias de SA, destacando aqueles cuja pessoa de referência tem atividade principal agrícola. Brasil: 2004, 2009 e 2013.

\begin{tabular}{l|r|r|r|r|r|r}
\hline \multirow{2}{*}{\begin{tabular}{l} 
Categorias de SA \\
\cline { 3 - 7 }
\end{tabular}} & \multicolumn{3}{|c|}{$\begin{array}{c}\text { Pessoa de referência com atividade } \\
\text { principal no setor agrícola }\end{array}$} & \multicolumn{3}{c}{ Demais domicílios particulares } \\
\cline { 2 - 7 } & 2004 & 2009 & 2013 & 2004 & 2009 & 2013 \\
\hline Com segurança alimentar & 53,7 & 61,1 & 63,3 & 67,2 & 71,1 & 79,2 \\
Insegurança leve & 20,9 & 21,3 & 21,9 & 17,5 & 18,3 & 13,9 \\
Insegurança moderada & 14,7 & 9,5 & 9,0 & 9,0 & 6,0 & 4,0 \\
Insegurança grave & 10,7 & 8,0 & 5,8 & 6,2 & 4,5 & 2,9 \\
\hline Fonte: Elaborado com base nos microdados da PNAD. & & & & & &
\end{tabular}

Note-se que o conjunto dos "Demais domicilios particulares" na Tabela 4 inclui, além daqueles cuja pessoa de referência tem atividade principal na indústria e nos serviços, aqueles cuja pessoa de referência não é ocupada, não podendo ser classificada conforme setor de atividade, como ocorre com aposentados e pensionistas.

Separar os domicílios agrícolas não constitui uma tentativa de reproduzir a classificação em rural e urbano. Trata-se de outro conceito. Na PNAD de 2013, $40,9 \%$ dos domicílios rurais não são agrícolas e um terço $(33,3 \%)$ dos domicílios agrícolas são urbanos.

A Tabela 4 mostra que, para os domicílios agrícolas, de 2009 a 2013, aumenta a proporção com segurança alimentar e diminui a proporção com insegurança moderada ou grave. É interessante notar que para os domicilios agrícolas o crescimento da proporção com segurança é mais intenso entre 2004 e 2009 e menos intenso entre 2009 e 2013, ocorrendo o inverso para os demais domicílios. Fica o desafio de explicar as causas de tal fenômeno.

\section{Determinantes da insegurança alimentar em 2013}

Na amostra da PNAD de 2013 há 116.466 domicílios particulares, todos com informação para sua classificação nas 4 categorias de segurança alimentar. Considerando o fator de expansão fornecido pelo IBGE, eles representam uma população de 65.257.741 domicílios.

Para desenvolver a análise dos determinantes da insegurança alimentar foi necessário eliminar da amostra os domicílios sem declaração de valor de qualquer das variáveis a serem utilizadas: a renda domiciliar, a cor, a idade e a escolaridade da pessoa de referência do domicilio e as características do domicilio usadas como variáveis explanatórias. Também foram excluídos os domićlios com pessoa de referência indígena e os com renda domiciliar nula. Passou-se a utilizar, então, uma amostra de 107.772 domićlios 
particulares, representando uma população de 60.411 mil domicílios, com 186.550 mil moradores ${ }^{3}$.

Vamos examinar, inicialmente, como a incidência de insegurança alimentar varia com a renda domiciliar per capita (RDPC), obtida dividindo a renda domiciliar pelo número de pessoas do domicílio, excluindo, no cálculo do numerador e do denominador, as pessoas cuja condição no domicilio é pensionista, empregado doméstico ou parente do empregado doméstico. São considerados 11 estratos de RDPC, com limites superiores dados por $10 \times 2^{k}$, com $k=1,2$, ..., 10, exceto no caso do último estrato, que inclui os domicílios com RDPC acima de $10 \times 2^{10}=\mathrm{R} \$ 10.240,00$. Há interesse em destacar estratos extremamente pobres, pois a insegurança alimentar está estreitamente associada à pobreza. A unidade monetária é a vigente em setembro de 2013, mês de referência da PNAD, quando o salário mínimo era igual a $\mathrm{R} \$ 678,00$. Os resultados estão na Tabela 5 .

Observa-se que todo tipo de insegurança alimentar tende a cair a partir do terceiro estrato (RDPC acima de $\mathrm{R} \$ 40)$. A insegurança alimentar grave cai sistematicamente com o aumento da RDPC já desde o primeiro estrato.

Como explicar o crescimento da proporção de domicílios com insegurança alimentar leve nos 3 primeiros estratos? Certamente é necessário levar em consideração o caráter parcialmente subjetivo da EBIA e que para famílias extremamente pobres a escassez de alimentos pode ter se tornado "usual", deixando de ser "registrada" e declarada.

Tabela 5. Porcentagem de domićlios com vários graus de insegurança alimentar, conforme estratos de renda domiciliar per capita. Brasil, 2013.

\begin{tabular}{|c|c|c|c|c|c|c|}
\hline \multirow[b]{2}{*}{ Estrato de RDPC (R\$) } & \multirow{2}{*}{$\begin{array}{c}\text { Domicílios } \\
\text { no estrato } \\
(1.000)\end{array}$} & \multicolumn{5}{|c|}{ Porcentagem com insegurança alimentar } \\
\hline & & Leve & Moderada & Grave & Total & $\begin{array}{c}\text { Moderada ou } \\
\text { grave }\end{array}$ \\
\hline Mais de 0 a 20 & 55 & 25,4 & 18,0 & 23,0 & 66,4 & 41,0 \\
\hline Mais de 20 a 40 & 342 & 28,2 & 15,3 & 19,1 & 62,6 & 34,4 \\
\hline Mais de 40 a 80 & 880 & 33,8 & 17,5 & 18,6 & 69,9 & 36,1 \\
\hline Mais de 80 a 160 & 2.120 & 33,6 & 15,7 & 12,7 & 62,1 & 28,5 \\
\hline Mais de 160 a 320 & 7.407 & 28,4 & 9,7 & 6,5 & 44,7 & 16,2 \\
\hline Mais de 320 a 640 & 14.977 & 19,6 & 5,5 & 3,2 & 28,4 & 8,7 \\
\hline Mais de 640 a 1.280 & 20.360 & 10,7 & 2,8 & 1,7 & 15,2 & 4,5 \\
\hline Mais de 1.280 a 2.560 & 9.289 & 5,6 & 0,9 & 0,5 & 7,1 & 1,5 \\
\hline Mais de 2.560 a 5.120 & 3.431 & 2,6 & 0,2 & 0,1 & 3,0 & 0,4 \\
\hline Mais de 5.120 a 10.240 & 1.206 & 1,4 & 0,1 & 0 & 1,5 & 0,1 \\
\hline Mais de 10.240 & 344 & 0 & 0 & 0 & 0 & 0 \\
\hline Total & 60.411 & 14,9 & 4,6 & 3,1 & 22,5 & 7,7 \\
\hline
\end{tabular}

3 Excluindo aqueles cuja condição na unidade domiciliar é pensionista, empregado doméstico ou parente de empregado doméstico, são 186.194 mil pessoas. 
Pode ser considerada estranha a existência de domicílios com RDPC acima de $\mathrm{R} \$ 2.560,00$ com insegurança alimentar grave. $\mathrm{Na}$ amostra de 107.772 domicilios há 9 , com 17 moradores, com RDPC acima de $\mathrm{R} \$ 2.560,00$ por mês e classificados como tendo insegurança alimentar grave. São poucos casos, que podem ser desconsiderados como dados estranhos que usualmente ocorrem em levantamentos com amostras tão grandes. Por outro lado, esses casos nos ajudam a lembrar do caráter parcialmente subjetivo da EBIA (e a natureza um tanto arbitrária das respostas). É caricatural o caso do domicílio mais rico entre aqueles com insegurança alimentar grave: uma única pessoa com rendimento mensal de $\mathrm{R} \$ 7.000,00$, cuja posição na ocupação é de empregador, 49 anos, branco, 12 anos de estudo, residente em domicílio com microcomputador ligado à internet, aparelho de DVD, carro e motocicleta, que respondeu "sim" às 8 perguntas da EBIA aplicáveis a seu caso (Ver Quadro 1).

Como já foi discutido em trabalhos anteriores [1,2], uma tabela de contingência simples, cruzando a classificação urbano-rural com as categorias de SA, mostra maior insegurança alimentar na área rural. Mas isso não significa que a situação rural do domicílio seja desfavorável à SA. Verifica-se, nos dados da PNAD de 2013, que os valores médios da RDPC e da escolaridade da pessoa de referência do domicílio são, na área rural, aproximadamente iguais à metade dos respectivos valores na área urbana. Basta controlar os efeitos da RDPC e da escolaridade para verificar que a localização em área rural é favorável à SA.

Em Hoffmann, 2013 [2], para captar o efeito da RDPC sobre a probabilidade de um domicilio apresentar insegurança alimentar, por meio de modelos de lógite, foram incluídas, como variáveis explanatórias, o logaritmo neperiano da RDPC, o seu quadrado e, eventualmente, o seu cubo. Esse modelo se mostrou satisfatório, mas ele impõe certa simetria nos efeitos da RDPC que não é apropriada nesse caso. Em uma parábola (polinômio de 2 o grau), por exemplo, o arco descendente é igual à imagem, em espelho plano, do arco ascendente. Visando aperfeiçoar a maneira de captar o efeito da RDPC, optamos por usar um modelo poligonal com 4 segmentos. Seja $P_{i}$ a probabilidade de ocorrer determinado tipo de insegurança alimentar em um domicílio com RDPC $=X_{i}$.
Vamos admitir que a relação em forma de poligonal entre o lógite

$$
Y_{i}=\ln \frac{P_{i}}{1-P_{i}}
$$

e o logaritmo da RDPC muda de inclinação quando $\ln X_{i}$ for igual a $\theta_{1}, \theta_{2}$ ou $\theta_{3}$. Sejam $Z_{h}$ (com $h=1,2,3)$ três variáveis binárias definidas de maneira que

$$
Z_{h}=0 \text { se } \ln X_{i} \leq \theta_{h}
$$

e

$$
Z_{h}=1 \text { se } \ln X_{i}>\theta_{h}
$$

Então, no que se refere à influência de $X_{i}$ sobre $P_{i}$, o modelo de lógite seria

$$
\begin{aligned}
Y_{i}=\alpha+\beta_{0} \ln X_{i} & +\beta_{1} Z_{1 i}\left(\ln X_{i}-\theta_{1}\right) \\
& +\beta_{2} Z_{2 i}\left(\ln X_{i}-\theta_{2}\right) \\
& +\beta_{3} Z_{3 i}\left(\ln X_{i}-\theta_{3}\right)
\end{aligned}
$$

Enquanto $\ln X_{i}$ não superar $\theta_{1}$, a relação entre $Y_{i}$ e $\ln X_{i}$ é uma reta com inclinação $\beta_{0}$. No ponto em que $\ln X_{i}=\theta_{1}$ há uma mudança de inclinação, que passa a ser $\beta_{0}+\beta_{1}$. Quando o logaritmo da RDPC está entre $\theta_{1}$ e $\theta_{3}$ aplica-se um terceiro segmento da poligonal, com declividade $\beta_{0}+\beta_{1}+\beta_{2}$. Finalmente, quando o logaritmo da RDPC supera $\theta_{3}$, passa a valer uma relação linear entre $Y_{i}$ e $\ln X_{i}$ com inclinação $\beta_{0}+\beta_{1}+\beta_{2}+\beta_{3}$.

Após algumas experiências, optamos por adotar como abscissas dos vértices da poligonal $\left(\theta_{h}\right)$ os valores $\theta_{1}=3, \quad \theta_{2}=5$ e $\theta_{3}=7$, correspondendo, aproximadamente, a valores das $\mathrm{RDPC}$ iguais a $\mathrm{R} \$ 20, \mathrm{R} \$ 148$ e $\mathrm{R} \$ 1.097$.

A tabela 6 mostra os resultados obtidos estimando um modelo de lógite para a probabilidade de um domicilio apresentar insegurança alimentar moderada ou grave. Excetuando-se a parte referente ao efeito da RDPC, as variáveis explanatórias incluídas são as mesmas utilizadas em artigo anterior utilizando os dados da PNAD de 2009 [2], e listadas no Apêndice A daquele artigo. As estimativas dos desvios padrões das estimativas dos parâmetros foram calculadas levando 
em consideração a estrutura complexa da amostra da PNAD 4 .

A Tabela 7 apresenta os resultados de um modelo igual para a probabilidade de um domicílio apresentar insegurança alimentar grave.

Nos dois casos utilizam-se dados de uma amostra de 107.772 domicílios. O coeficiente de concordância $(c)$, que é uma medida da qualidade do ajustamento do modelo de lógite aos dados baseada no número de pares concordantes e pares discordantes, é 0,820 para a Tabela 6 e é 0,836 para a Tabela 7 .

Ao analisar o efeito da RDPC, é essencial lembrar que já no segundo estrato (acima de $\mathrm{R} \$ 20$ ) a declividade da função é dada por uma soma de coeficientes, como explicado anteriormente. Tanto na Tabela 6 como na Tabela 7 as 3 somas são negativas e estatisticamente diferentes de zero (probabilidade caudal do teste inferior a $0,1 \%$ ).

Tabela 6. Modelo de lógite para a probabilidade de um domicílio ter insegurança alimentar moderada ou grave. Brasil, 2013.

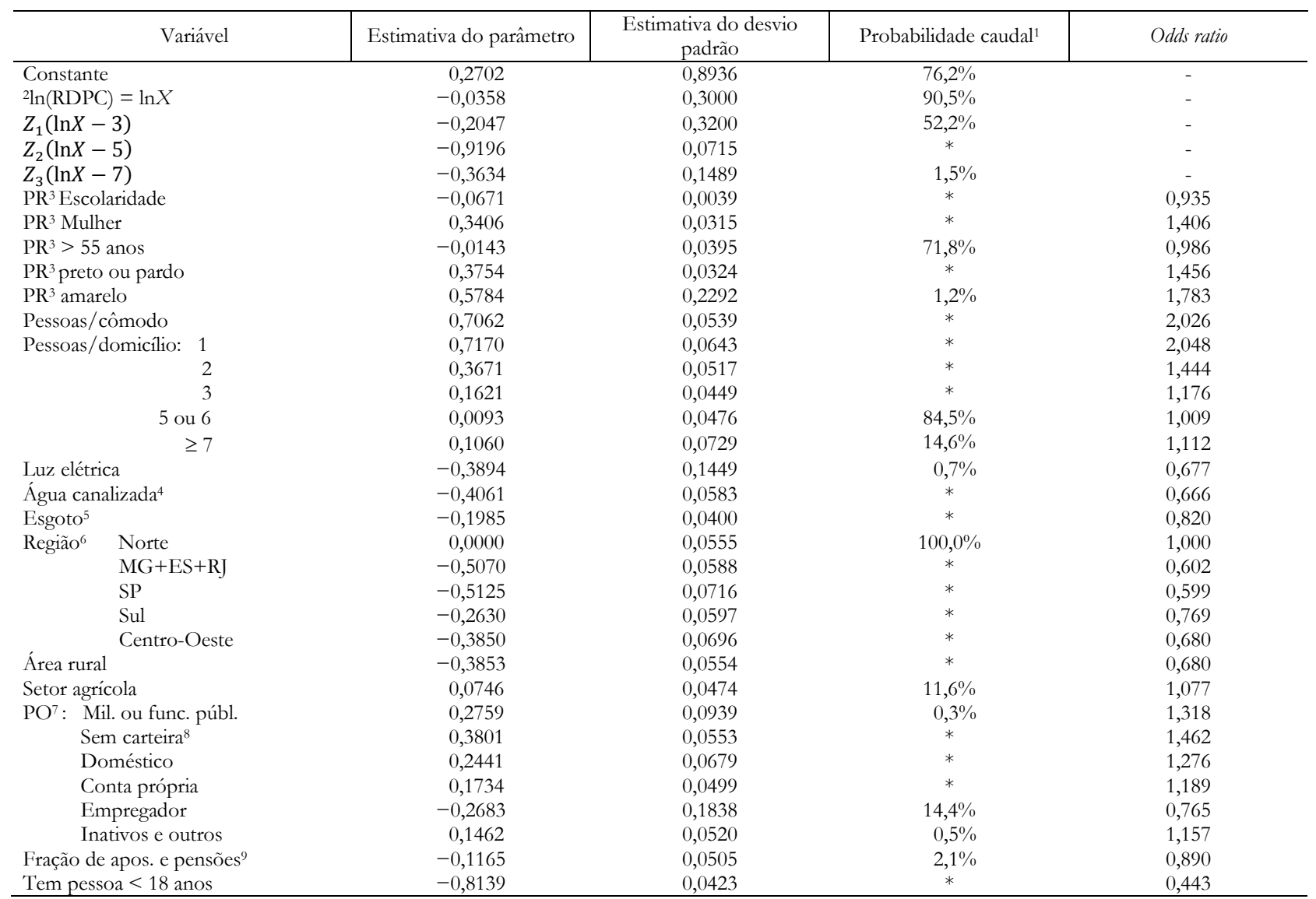

${ }^{1}$ Probabilidade caudal do teste da hipótese de nulidade do parâmetro. O asterisco assinala os casos em que essa probabilidade é inferior a $0,1 \%$.

2 Logaritmo neperiano da renda domiciliar per capita (RDPC).

${ }_{3}^{3}$ Pessoa de referência do domicílio, adotando como base o homem branco com até 55 anos de idade.

4 Tem água canalizada em pelo menos um cômodo.

${ }^{5}$ Escoadouro do banheiro ou sanitário ligado a rede coletora e/ou fossa séptica.

${ }^{6}$ A Região Nordeste é adotada como base.

${ }^{7}$ Posição na ocupação da pessoa de referência do domicílio, adotando como base os empregados com carteira.

8 Empregados sem carteira ou sem declaração de carteira.

${ }^{9}$ Fração da renda domiciliar constituída por aposentadorias e pensões pagas pelo poder público.

${ }^{4}$ Utilizou-se o procedimento SURVEYLOGISTIC do Statistical Analysis System (SAS). 
Brasil, 2013: mais segurança alimentar. Hoffmann.

Tabela 7. Modelo de lógite para a probabilidade de um domicílio ter insegurança alimentar grave. Brasil, 2013.

\begin{tabular}{|c|c|c|c|c|c|}
\hline & Variável & Estimativa do parâmetro & $\begin{array}{c}\text { Estimativa do desvio } \\
\text { padrão }\end{array}$ & Probabilidade caudal ${ }^{1}$ & Odds ratio \\
\hline \multicolumn{2}{|c|}{ Constante } & $-0,0969$ & 1,0767 & $92,8 \%$ & - \\
\hline \multicolumn{2}{|c|}{${ }^{2} \ln (\mathrm{RDPC})=\ln X$} & $-0,1380$ & 0,3568 & $69,9 \%$ & - \\
\hline \multicolumn{2}{|c|}{$Z_{1}(\ln X-3)$} & $-0,1946$ & 0,3760 & $60,5 \%$ & - \\
\hline \multicolumn{2}{|c|}{$Z_{2}(\ln X-5)$} & $-0,8887$ & 0,0883 & * & - \\
\hline \multicolumn{2}{|c|}{$Z_{3}(\ln X-7)$} & $-0,2292$ & 0,2446 & $34,9 \%$ & - \\
\hline \multicolumn{2}{|c|}{$\mathrm{PR}^{3}$ Escolaridade } & $-0,0840$ & 0,0060 & * & 0,919 \\
\hline \multicolumn{2}{|c|}{$\mathrm{PR}^{3}$ Mulher } & 0,2479 & 0,0466 & * & 1,281 \\
\hline \multicolumn{2}{|c|}{$\mathrm{PR}^{3}>55$ anos } & $-0,0572$ & 0,0616 & $35,3 \%$ & 0,944 \\
\hline \multicolumn{2}{|c|}{$\mathrm{PR}^{3}$ preto ou pardo } & 0,3359 & 0,0494 & * & 1,399 \\
\hline \multicolumn{2}{|c|}{$\mathrm{PR}^{3}$ amarelo } & 0,2970 & 0,3721 & $42,5 \%$ & 1,346 \\
\hline \multicolumn{2}{|c|}{ Pessoas/cômodo } & 0,5835 & 0,0603 & * & 1,792 \\
\hline & 1,1354 & 0,0919 & * & 3,113 \\
\hline \multicolumn{2}{|c|}{2} & 0,4696 & 0,0757 & * & 1,599 \\
\hline \multirow{2}{*}{\multicolumn{2}{|c|}{$\begin{array}{r}3 \\
5 \text { ou } 6\end{array}$}} & 0,2076 & 0,0680 & $0,2 \%$ & 1,231 \\
\hline & & 0,1653 & 0,0721 & $2,2 \%$ & 1,180 \\
\hline \multicolumn{2}{|r|}{$\geq 7$} & 0,2046 & 0,0990 & $3,9 \%$ & 1,227 \\
\hline \multicolumn{2}{|c|}{ Luz elétrica } & $-0,6157$ & 0,1714 & * & 0,540 \\
\hline \multicolumn{2}{|c|}{ Água canalizada ${ }^{4}$} & $-0,5374$ & 0,0801 & * & 0,584 \\
\hline \multicolumn{2}{|c|}{ Esgoto $^{5}$} & $-0,1357$ & 0,0537 & $1,1 \%$ & 0,873 \\
\hline \multirow[t]{5}{*}{ Região $^{6}$} & Norte & 0,2524 & 0,0734 & * & 1,287 \\
\hline & $\mathrm{MG}+\mathrm{ES}+\mathrm{RJ}$ & $-0,2982$ & 0,0813 & * & 0,742 \\
\hline & SP & $-0,0604$ & 0,0927 & $51,5 \%$ & 0,941 \\
\hline & Sul & 0,0432 & 0,0954 & $65,0 \%$ & 1,044 \\
\hline & Centro-Oeste & $-0,1179$ & 0,0847 & $16,4 \%$ & 0,889 \\
\hline \multicolumn{2}{|c|}{ Área rural } & $-0,5178$ & 0,0793 & $*$ & 0,596 \\
\hline \multicolumn{2}{|c|}{ Setor agrícola } & $-0,0725$ & 0,0729 & $32,0 \%$ & 0,930 \\
\hline \multirow{6}{*}{$\mathrm{PO}^{7}:$} & il. ou func. públ. & 0,1031 & 0,1644 & $53,1 \%$ & 1,109 \\
\hline & n carteira ${ }^{8}$ & 0,4651 & 0,0880 & * & 1,592 \\
\hline & méstico & 0,3571 & 0,1015 & $*$ & 1,429 \\
\hline & nta própria & 0,2138 & 0,0818 & $0,9 \%$ & 1,238 \\
\hline & pregador & $-1,1485$ & 0,4547 & $1,2 \%$ & 0,317 \\
\hline & tivos e outros & 0,2955 & 0,0835 & * & 1,344 \\
\hline \multicolumn{2}{|c|}{ Fração de apos. e pensões ${ }^{9}$} & $-0,1927$ & 0,0791 & $1,5 \%$ & 0,825 \\
\hline \multicolumn{2}{|c|}{ Tem pessoa $<18$ anos } & $-0,6839$ & 0,0643 & * & 0,505 \\
\hline
\end{tabular}

${ }_{1}^{1}$ Probabilidade caudal do teste da hipótese de nulidade do parâmetro. $\mathrm{O}$ asterisco assinala os casos em que essa probabilidade é inferior a $0,1 \%$.

${ }^{2}$ Logaritmo neperiano da renda domiciliar per capita (RDPC).

${ }^{3}$ Pessoa de referência do domicílio, adotando como base o homem branco com até 55 anos de idade.

${ }^{4}$ Tem água canalizada em pelo menos um cômodo.

${ }^{5}$ Escoadouro do banheiro ou sanitário ligado a rede coletora e/ou fossa séptica.

${ }^{6}$ A Região Nordeste é adotada como base.

7 Posição na ocupação da pessoa de referência do domicílio, adotando como base os empregados com carteira.

${ }^{8}$ Empregados sem carteira ou sem declaração de carteira.

${ }^{9}$ Fração da renda domiciliar constituída por aposentadorias e pensões pagas pelo poder público.

Deixamos de apresentar pormenorizadamente os resultados obtidos com um modelo de lógite para a probabilidade de ocorrer insegurança alimentar de qualquer grau. A Figura 1 ilustra como o lógite estimado em cada caso varia com o logaritmo da RDPC e a Figura 2 mostra como varia, também em função do logaritmo da RDPC, a correspondente probabilidade de o domicílio pertencer a uma das três categorias consideradas: insegurança alimentar de qualquer grau, insegurança moderada ou grave e apenas insegurança grave. Para obter os valores estimados, considerou-se um domicílio na área urbana da Região Nordeste, com 4 pessoas, 0,7 pessoas por cômodo, $20 \%$ da renda domiciliar proveniente de aposentadorias e pensões pagas pelo poder público, sem pessoa menor de 18 anos, com luz elétrica, água canalizada e esgoto apropriado e cuja pessoa de referência é um homem preto ou pardo, com idade igual ou inferior a 55 anos, 7 anos de escolaridade, emprego com carteira fora do setor agrícola. 
Figura 1. A variação do lógite $(Y)$ com o logaritmo da RDPC nos modelos estimados para insegurança alimentar (total, moderada ou grave, grave) no Brasil, 2013.

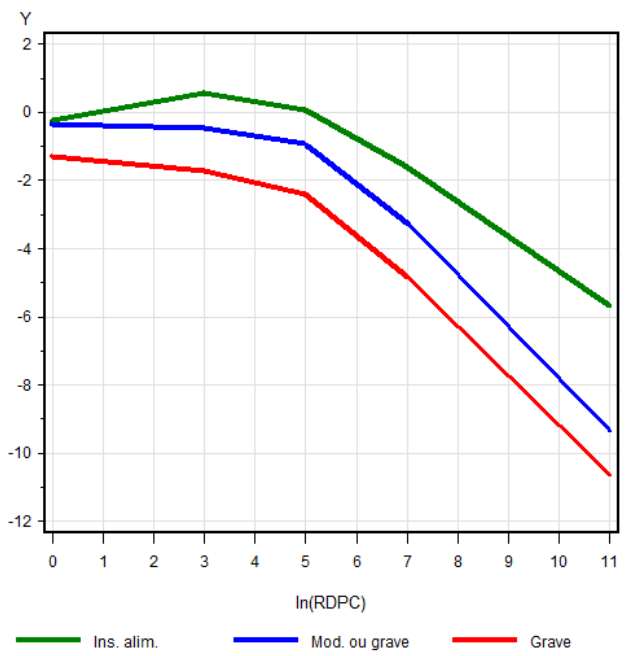

Figura 2. Variação da porcentagem de domicílios com insegurança alimentar (total, moderada ou grave, grave) em função do logaritmo da RDPC conforme modelos de lógite estimados. Brasil, 2013.

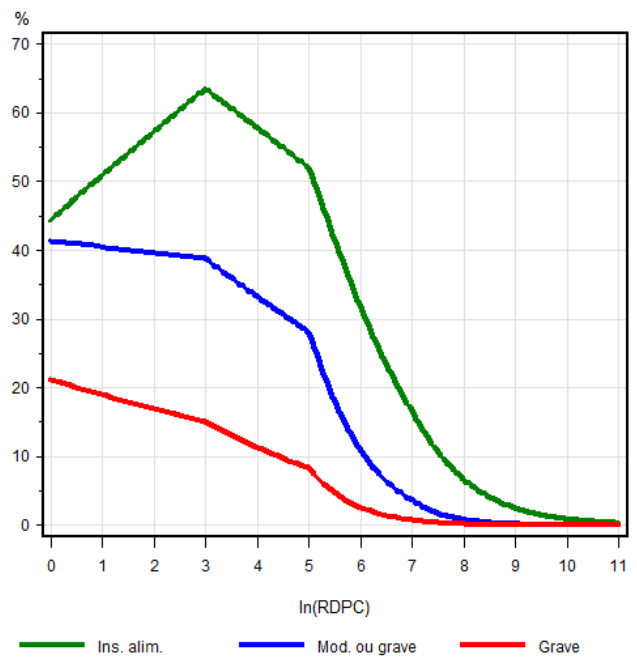

Observa-se, nas Figuras 1 e 2, que o valor de $Y$ e a probabilidade estimada de insegurança alimentar leve, moderada ou grave crescem com a RDPC até o ponto em que o logaritmo da RDPC é igual a 3, isto é, até uma RDPC de R $\$ 20$ mensais. Trata-se, aqui, do efeito da RDPC depois de controlados os efeitos de todas as demais variáveis explanatórias do modelo. Como a lógica econômica é que a insegurança alimentar deve diminuir com o aumento da renda, essa relação positiva entre as duas variáveis, para domicilios extremamente pobres provavelmente seja devida ao caráter parcialmente subjetivo da EBIA. Cabe lembrar, também, que as informações sobre renda são menos confiáveis nas caudas da distribuição.

Todos os demais segmentos de reta na Figura 1 são decrescentes, indicando, como esperado, que a insegurança alimentar diminui com o aumento da RDPC. Verifica-se, na Figura 2, que a probabilidade estimada de qualquer categoria de insegurança 
alimentar se torna praticamente nula quando a RDPC ultrapassa $\exp (10)$, aproximadamente 22 mil reais. A probabilidade estimada de insegurança grave já se torna praticamente nula para valores da RDPC acima de $\exp (7)$, cerca de $\mathrm{R} \$ 1.100,00$.

A Tabela 6 mostra, em geral, resultados que confirmam os efeitos já observados na Tabela 10 (p. 235) de Hoffmann, 2013 [2], com os resultados de um modelo de lógite semelhante estimado com dados da PNAD de 2009. Considerando apenas coeficientes fortemente significativos verifica-se que, controlados os efeitos das demais variáveis, a probabilidade de insegurança alimentar moderada ou grave:

a) diminui com o crescimento da RDPC;

b) diminui com o aumento da escolaridade da pessoa de referência;

c) aumenta quando a pessoa de referência é mulher;

d) aumenta quando a pessoa de referência é preta ou parda;

e) aumenta com o número de pessoas por cômodo;

f) é maior em domicílios com apenas 1 ou 2 pessoas;

g) é menor em domicílios com luz elétrica, água canalizada e esgoto adequado;

h) é menor no Sudeste, no Sul e Centro-Oeste, em comparação com o Nordeste;

i) é menor na área rural;

j) em comparação com os empregados com carteira, é maior para empregados sem carteira, empregados domésticos ou trabalhadores por conta própria;

k) é menor nos domicilios nos quais há pessoa menor de 18 anos.

Há poucos casos de coeficientes que mudam de sinal. Na Tabela 6 o coeficiente da variável binária que indica que a pessoa de referência se declarou amarela é positivo e significativo a $5 \%$. Mas com os dados de 2009 havíamos obtido um coeficiente negativo, mas não significativo, para a mesma variável. $\mathrm{Na}$ Tabela 7, referente ao modelo de lógite para insegurança alimentar grave, o coeficiente dessa variável é positivo, mas não significativo. Parece razoável concluir que, depois de controlados os efeitos das demais variáveis, não há efeito de ser "amarelo" sobre a probabilidade de insegurança alimentar.
O coeficiente para a variável binária que indica que a pessoa de referência do domicílio é militar ou funcionário público estatutário é positivo e significativo quando são usados os dados da PNAD de 2013, como mostra a Tabela 6 , mas era praticamente nulo quando estimado com os dados de 2009. Na Tabela 7, no modelo para insegurança grave, o coeficiente dessa variável não é estatisticamente diferente de zero. A conclusão cautelosa é que, controlados os efeitos das demais variáveis explanatórias, não se constata efeito de a pessoa de referência ser militar ou funcionário público estatutário sobre a probabilidade de o domicilio ter insegurança alimentar. $\mathrm{Na}$ ausência de controle dos efeitos das demais variáveis o efeito é negativo e significativo, já que essa categoria tem RDPC e escolaridade elevadas.

Tanto na Tabela 6 como na Tabela 7 o efeito de o domicílio estar na área rural é negativo e fortemente significativo. Entretanto, sem o controle do efeito das demais variáveis explanatórias, o efeito de "rural" é positivo e significativo, isto é, a insegurança alimentar é maior na área rural. Basta controlar os efeitos da RDPC e da escolaridade da pessoa de referência para obter a mudança no sinal do efeito de "rural". Isso se explica pelo fato de tanto a RDPC como a escolaridade serem muito menores para domicílios rurais do que para domicilios urbanos $(\mathrm{R} \$ 502,8$ versus $\mathrm{R} \$ 1.036,8$ e 4,0 versus 8,1 anos de estudo).

Outro caso da mudança de sinal do coeficiente devido à introdução de controles é o da variável binária que indica a presença, no domicilio, de pessoa menor de 18 anos. Em uma análise sem controles observa-se que a insegurança alimentar é estatisticamente maior quando há pessoa menor de 18 anos. Basta controlar o efeito da RDPC para que o coeficiente para a presença de pessoa menor de 18 anos se torne negativo e estatisticamente significativo, pois essa presença está associada a uma menor RDPC. Depois de considerar o efeito de todas as demais variáveis listadas nas Tabelas 6 e 7 verifica-se que o efeito daquela variável binária permanece negativo e fortemente significativo. Note-se que a presença de pessoa menor de 18 anos também está associada a mais pessoas por cômodo e mais pessoas por domicílio ${ }^{5}$. 
Há também coeficientes que não mudam de sinal, mas cuja magnitude se altera substancialmente com a introdução de controles. Quando, por exemplo, se considera exclusivamente o efeito da localização regional, a odds ratio para insegurança alimentar moderada ou grave de localização no Estado de São Paulo em comparação com localização no Nordeste é 0,217. Na Tabela 6 a odds ratio para localização no Estado de São Paulo é 0,599, permanecendo estatisticamente abaixo de 1 . A mudança na magnitude do efeito de estar no Estado de São Paulo se deve, em parte, ao fato de essa região ter RDPC média mais alta ( $\mathrm{R} \$ 1.234,1$ contra $\mathrm{R} \$ 608,3$ no Nordeste) e escolaridade média da pessoa de referência mais alta ( 8,6 contra 6,0 anos de estudo no Nordeste).

\section{Modelos de lógite estimados agregando os dados de 2009 e 2013}

Da PNAD de 2013, após as depurações, temos uma amostra de 107.772 observações. Analogamente, da PNAD de 2009 obtemos uma amostra de 115.260 observações. Empilhando os dois conjuntos, obtemos uma amostra de 223.032 observações. Criando uma variável binária "ano" com valor zero em 2009 e valor 1 em 2013, estimamos modelos de lógite com todas as variáveis listadas nas Tabelas 6 ou 7 , considerando como variável dependente a probabilidade de ocorrer insegurança alimentar de qualquer grau, insegurança moderada ou grave ou, ainda, apenas insegurança grave. Deixamos de apresentar os resultados pormenorizados, que ocupariam muito espaço. Os coeficientes de qualidade do ajustamento são, respectivamente, 0,775 , 0,824 e 0,840 . Os coeficientes da variável binária para "ano" são sempre negativos e fortemente significativos (probabilidade caudal do teste de nulidade do parâmetro inferior a $0,1 \%$ ), indicando que a redução da insegurança alimentar é maior do que a que, por meio do modelo, pode ser explicada por variações nas variáveis explanatórias entre os dois anos como, por exemplo, o aumento da escolaridade média da pessoa de referência de 7,0 anos em 2009 para 7,5 anos em 2013.

Ao agregar os dados de 2009 e 2013, os valores da RDPC do primeiro ano foram colocados na mesma unidade monetária dos dados do segundo ano, usando como deflator o Índice Nacional de Preços ao Consumidor (INPC). Verifica-se que, para as amostras analisadas, a RDPC média cresce de R\$802 em 2009 para $\mathrm{R} \$ 954$ em 2013. Mas houve, também, uma redução da desigualdade da distribuição da renda que certamente contribuiu para diminuir a insegurança alimentar. Para a amostra analisada, o índice de Gini da distribuição da RDPC cai de 0,536 em 2009 para 0,519 em 2013.

\section{Atitude adotada quando faltou alimento}

Na PNAD de 2013 há duas perguntas novas relativas à segurança alimentar. Nos microdados, a variável V2138 apresenta códigos para as 15 alternativas de resposta à pergunta sobre a "Principal atitude adotada quando faltou alimento":

1. Pediram alimentos emprestados a parentes, vizinhos e/ou amigos.

2. Prestaram pequenos serviços a parentes e amigos em troca de alimentos.

3. Compraram fiado.

4. Deixaram de comprar alimentos supérfluos (biscoitos, refrigerantes, ...).

5. Comeram menos carnes.

6. Comeram menos frutas, verduras e legumes.

7. Foram pescar, caçar e colher frutos.

8. Xeparam ${ }^{6}$ em feiras e mercados e aproveitaram alimentos.

9. Procuraram emprego (emprego melhor ou um segundo emprego; pequenos serviços; um membro da família que não trabalhava arrumou emprego etc.).

10. Receberam ajuda de associação religiosa, igreja.

11. Receberam ajuda do governo municipal, estadual, federal.

12. Receberam alimentos da comunidade, vizinhos, parentes e amigos.

13. Cadastraram-se em algum programa governamental de assistência social.

14. Não fizeram nada.

15. Pediram dinheiro emprestado.

A variável V2139 apresenta o código da resposta à pergunta sobre "Outra atitude adotada quando faltou alimento". As 15 alternativas são exatamente as mesmas que para a variável V2138.

Para as duas variáveis há, na amostra, muitos domicílios sem resposta, que incluem tanto os casos em que as perguntas não se aplicam (porque o domicílio tem segurança alimentar) como casos em que o entrevistado não respondeu. Para a variável V2138 há

${ }^{6}$ No google consta que xepa significa "frutas, legumes, verduras etc. que não foram vendidos na feira livre e os feirantes deixam na rua e são coletados pelas pessoas". 
resposta para todos os domicílios classificados como tendo insegurança grave, para $77 \%$ dos domicílios da amostra com insegurança moderada e para apenas $22 \%$ dos com insegurança leve. Optamos por passar a analisar apenas a amostra de 7.571 domicílios com insegurança alimentar moderada ou grave e com resposta na variável V2138. Essa amostra representa uma população de 4.005 mil domicílios.

A Tabela 8 mostra a distribuição das respostas à primeira pergunta (V2138) pelos 15 tipos na população de domicílios, para os com insegurança moderada, com insegurança grave e agregando as duas categorias. É notória a grande frequência das respostas 3 (Compraram fiado) e 1 (Pediram alimentos emprestados a parentes, vizinhos e/ou amigos) ${ }^{7}$.

Verificamos que a distribuição das respostas pelas 15 alternativas difere bastante para domicilios urbanos e domicilios rurais. Como é de se esperar, a opção 7 (pescar, caçar e colher frutos) é muito mais comum para domicílios rurais $(4,6 \%)$ do que para os urbanos $(0,6 \%)$. A alternativa 3 (compraram fiado) é mais usual na área rural $(55,7 \%)$ do que na urbana
$(36,2 \%)$, ocorrendo o inverso com a alternativa 15 (pediram dinheiro emprestado): 5,7\% para domicílios urbanos e 1,9\% para os rurais.

Analisando como as respostas na variável V2138 variam com o nível de RDPC, é interessante notar que as proporções de respostas 4 e 5 tendem a crescer com a renda. A possível interpretação é que os muito pobres já não têm consumo de alimentos supérfluos ou carne que possa ser reduzido. A proporção de respostas 1 diminui com o aumento da renda e a proporção de respostas 3 também mostra certa tendência decrescente em função da RDPC.

$\mathrm{Na}$ variável V2139 as respostas mais frequentes são 1, 3, 4 e 14. Considerando as duas respostas, observa-se que as combinações mais frequentes (mais de 10\% do total considerado) são 1 com 3 e 3 com 1 . Destacam-se, ainda, com frequência acima de $4 \%$ do total, as combinações $3 \mathrm{com} 4,3 \mathrm{com}$ 5, 1 com 14, 3 com 14 e 3 com 15.

Tabela 8. Distribuição dos domicílios com insegurança alimentar moderada ou grave conforme a atitude adotada quando faltou alimento.

\begin{tabular}{|c|c|c|c|}
\hline \multirow{2}{*}{ Código da resposta ${ }^{1}$} & \multicolumn{3}{|c|}{ \% do tipo de atitude para domicílios com insegurança alimentar } \\
\hline & Moderada & Grave & Moderada ou grave \\
\hline 1. Pediram alimentos emprestados & 24,7 & 34,0 & 29,1 \\
\hline 2. Prestaram pequenos serviços por alimentos & 2,5 & 3,2 & 2,8 \\
\hline 3. Compraram fiado & 45,7 & 36,1 & 41,2 \\
\hline 4. Deixaram de comprar alimentos supérfluos & 6,6 & 3,8 & 5,3 \\
\hline 5. Comeram menos carnes & 2,9 & 2,7 & 2,8 \\
\hline 6. Comeram menos frutas, verduras e legumes & 1,0 & 0,8 & 0,9 \\
\hline 7. Foram pescar, caçar e colher frutos & 1,4 & 1,9 & 1,6 \\
\hline 8. Xeparam e aproveitaram alimentos & 0,1 & 0,5 & 0,3 \\
\hline 9. Procuraram emprego & 1,8 & 2,2 & 2,0 \\
\hline 10. Receberam ajuda de associação religiosa & 1,1 & 1,4 & 1,2 \\
\hline 11. Receberam ajuda do governo & 0,4 & 1,0 & 0,7 \\
\hline 12. Receberam alimentos da comunidade/amigos & 2,8 & 4,2 & 3,5 \\
\hline 13. Cadastraram-se em programa de assistência & 0,3 & 0,5 & 0,4 \\
\hline 14. Não fizeram nada & 3,6 & 3,4 & 3,5 \\
\hline 15. Pediram dinheiro emprestado & 5,1 & 4,3 & 4,7 \\
\hline
\end{tabular}

Fonte: Elaborada a partir dos microdados da PNAD de 2013.

1 Ver no texto a descrição completa de cada tipo de resposta.

${ }^{7}$ Como curiosidade, registre-se que aquele domicílio com insegurança alimentar grave e RDPC igual a R\$7.000 optou pela resposta 14 (Não fizeram nada). 


\section{CONSIDERAÇÕES FINAIS}

A análise dos dados da PNAD de 2013 confirma que a insegurança alimentar está fortemente associada a baixos níveis de RDPC, com tendência de maior insegurança quando a fonte de renda é instável. A maior escolaridade da pessoa de referência do domicílio e a existência de luz elétrica, água encanada e esgoto adequado também contribuem de maneira relevante para diminuir a insegurança alimentar.

A insegurança alimentar medida pela EBIA é um complemento a outras medidas da qualidade de vida, mas não deve ser considerada um substituto para uma boa medida da renda. Não se deve esquecer o caráter parcialmente subjetivo das respostas às 14 perguntas que são a base da escala.

Mesmo considerando as limitações da EBIA, os dados da PNAD de 2004, 2009 e 2013 deixam claro que ocorreu redução da insegurança alimentar no Brasil, como mostrou o próprio IBGE [3]. Modelos de lógite estimados agregando os dados de 2009 e 2013 mostram que todas as variáveis incluídas não explicam integralmente a redução da insegurança alimentar de 2009 a 2013. Além do aumento da renda média, há efeito favorável de mudança na forma da distribuição da renda, com redução da desigualdade. Para isso certamente tiveram papel importante as transferências de renda (Bolsa Família, Benefício da Prestação Continuada, aposentadorias rurais, etc.) e o crescimento do valor real do salário mínimo. Mas há outros fatores condicionando as mudanças na distribuição da renda no Brasil, como discutido em Hoffmann, 2014 [8].

\section{REFERÊNCIAS}

[1] Hoffmann R. Determinantes da insegurança alimentar no Brasil: análise dos dados da PNAD de 2004. Segur Aliment Nutr. 2008;15(1):49-61.

[2] Hoffmann R. Determinantes da insegurança alimentar no Brasil em 2004 e 2009. Segur Aliment Nutr. 2013;20(2):21935.

[3] Instituto Brasileiro de Geografia e Estatística. Pesquisa Nacional por Amostra de Domicilios: segurança alimentar 2013. Rio de Janeiro: Instituto Brasileiro de Geografia e Estatística; 2014. 123 p.

[4] Instituto Brasileiro de Geografia e Estatística. Pesquisa Nacional por Amostra de Domicilios: segurança alimentar 2004/2009. Rio de Janeiro: Instituto Brasileiro de Geografia e Estatística; 2010. 171 p.

[5] Instituto Brasileiro de Geografia e Estatística. Pesquisa Nacional por Amostra de Domicilios: segurança alimentar 2004. Rio de Janeiro: Instituto Brasileiro de Geografia e Estatística; 2006. 127 p.

[6] Segall-Corrêa AM, Marin-Leon L. A segurança alimentar no Brasil: proposição e usos da escala brasileira de medida da insegurança alimentar (EBIA) de 2003 a 2009. Segur Aliment Nutr. 2009;16(2):1-19.

[7] Pérez-Escamilla R, Segall-Corrêa AM. Indicadores e medidas de insegurança alimentar. Rev Nutr. 2008;21 Supl;15-26.

[8] Hoffmann R. Transferências de renda e desigualdade no Brasil (1995-2011). In: Campello T, Neri M, editores. Programa Bolsa Família: uma década de inclusão e cidadania. Brasilia: Instituto de Pesquisa Econômica Aplicada; 2013. p. 207-16. 
APÊNDICE A. A construção da variável-síntese para segurança alimentar nos microdados.

Nas publicações do IBGE [3,4,5] há explicações pormenorizadas sobre a aplicação da EBIA nas pesquisas suplementares sobre segurança alimentar. Entretanto, como esses esclarecimentos são dirigidos ao leitor geral, algumas vezes não estão na forma mais apropriada para os pesquisadores que usam os microdados. Os comentários complementares a seguir podem ser úteis para tais pesquisadores.

No arquivo de microdados da PNAD de 2013 a variável-síntese para segurança alimentar é a V4623, classificando o domicílio em cada uma das 8 categorias descritas na Tabela 1. Essa variável pode ser reproduzida fazendo-se a soma das respostas "sim" nas variáveis correspondentes às 14 perguntas do Quadro 1: V2103, V2105, V2107, V2109, V2113, V2115, V2117, V2121, V2124, V2126, V2128, V2130, V2132 e V2134.

No arquivo de microdados da PNAD de 2009 a variável-síntese de segurança alimentar que deve ser usada é a V4744. Ela é baseada no número de respostas "sim" nas mesmas 14 variáveis usadas em 2013. Notase, entretanto, que nos microdados há resultados para duas perguntas (V2111 e V2119) que não foram usadas na construção da variável-síntese de 2009. Essas duas variáveis são "resíduos" das perguntas usadas em 2004.

Na PNAD de 2004 a construção da variávelsíntese (V4623 ou V4744) foi feita de maneira um pouco diferente. Foram consideradas 16 perguntas, cujas respostas foram registradas em V2103, V2105, V2107, V2109, V2111, V2113, V2115, V2117, V2119, V2121, V2124, V2126, V2128, V2130, V2132 e V2134. As respostas V2111 e V2113 foram agregadas, contando-se 1 ponto se a resposta fosse "sim" em uma delas ou nas duas. Dessa maneira, o número de pontos variava de zero a 15 e a classificação final de cada domicílio foi feita de acordo com a Tabela A1.

Tabela A1. Pontuação para classificação dos domicílios, com e sem menores de 18 anos, usada originalmente no arquivo de microdados da PNAD de 2004.

\begin{tabular}{l|c|c}
\hline \multirow{2}{*}{ Classificação } & \multicolumn{2}{|c}{ Número de pontos para domicílio } \\
\cline { 2 - 3 } & Com menores de 18 anos & Sem menores de 18 anos \\
\hline Segurança alimentar & 0 & 0 \\
Insegurança alimentar leve & 1 a 5 & 1 a 3 \\
Insegurança alimentar moderada & 6 a 10 & 4 a 6 \\
Insegurança alimentar grave & 11 a 15 & 7 a 9 \\
\hline
\end{tabular}

Ao fazer comparações de 2004 com 2009 e 2013 o IBGE [3,4] reclassifica os domicilios em 2004, desprezando as variáveis V2111 e V2119. Restam 14 perguntas e o número de respostas "sim" determina a classificação do domicílio, conforme a Tabela 1. O pesquisador que usa os microdados deve fazer essa reclassificação com base nas 14 variáveis, pois no arquivo de microdados da PNAD de 2004 as variáveis- síntese (V4623 e V4744) continuam definidas conforme o procedimento original ${ }^{8}$. Fazendo isso, é possível reproduzir, a partir dos microdados, os resultados apresentados na Tabela 3 da última publicação do IBGE sobre o tema [3].

${ }^{8}$ Infelizmente isso não foi feito em artigo anterior [2], no qual foram usadas as variáveissíntese dos microdados de 2004 e 2009. 